\title{
Ocular Signs Caused by Dural Arteriovenous Fistula without Involvement of the Cavernous Sinus: A Case Series with Review of the Literature
}

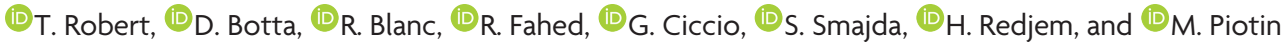

\begin{abstract}
SUMMARY: Carotid cavernous fistula is a well-known clinical and angiographic entity responsible for ocular signs and symptoms. On the contrary, ocular signs are unusual in the presentation of cranial dural arteriovenous fistulas at locations other than the cavernous sinus. We retrospectively analyzed data focusing on the pathophysiology of ophthalmologic signs and their angiographic explanations. Thirteen patients were included with a mean age of 50 years. The most common signs were chemosis (61.5\%), loss of visual acuity (38.5\%), exophthalmia (38.5\%), and ocular hypertension (7.7\%). Dural arteriovenous fistulas presenting with ocular signs could be classified into 4 types due to their pathologic mechanism (local venous reflux into the superior ophthalmic vein, massive venous engorgement of the cerebrum responsible for intracranial hypertension, compression of an oculomotor nerve by a venous dilation, or intraorbital fistula with drainage into the superior ophthalmic vein).
\end{abstract}

ABBREVIATIONS: $\mathrm{dAVF}=$ dural arteriovenous fistula; $\mathrm{SOV}=$ superior ophthalmic vein

C arotid-cavernous fistula is a well-known clinical and angiographic entity responsible for ocular signs and symptoms. ${ }^{1,2}$ The venous engorgement transmitted from the cavernous sinus to the superior and/or, rarely, the inferior ophthalmic veins could explain these ocular signs. On the contrary, ocular signs are very unusual in the presentation of cranial dural arteriovenous fistulas (dAVFs) in locations other than the cavernous sinus. ${ }^{3}$ In the literature, isolated cases of dAVFs without involvement of the cavernous sinus, revealed by ocular signs, could be found. ${ }^{4,5}$ These particular and welldescribed cases were rarely accompanied by a pathophysiologic explanation of the clinical signs. The authors categorized the different dAVFs associated with ocular signs on the basis of a 15-year-experience in the endovascular treatment of cranial dAVFs.

\section{MATERIALS AND METHODS \\ Patient Selection}

We have maintained an ongoing prospective data base with demographic, clinical, and angiographic information regarding patients with cranial dAVFs. From 2000 to 2015, 305 patients with

Received February 2, 2016; accepted after revision March 31.

From the Department of Interventional Neuroradiology, Rothschild Foundation Hospital, Paris, France.

T. Robert and D. Botta have participated equally in this work.

Please address correspondence to Thomas Robert, MD, Rothschild Foundation Hospital, 25 Rue Manin, 75019 Paris, France; e-mail: thomas.robert43@gmail.com

http://dx.doi.org/10.3174/ajnr.A4831
dAVFs have been evaluated in our institution. In this study, we retrospectively reviewed data of patients who met the following criteria: 1) the first clinical sign was ophthalmic, 2) a cranial dAVF was confirmed by digital subtraction angiography, and 3) the location of the fistulous point of the dAVF was not in the cavernous sinus. Demographic data were recorded for each patient, including age, sex, vascular risk factors, and clinical presentation.

\section{Pretherapeutic Clinical Evaluation}

A clinical history and a neurologic examination were performed for each patient before the treatment of the fistula. We mainly looked for the presence of an etiology, the duration of symptoms, the type of clinical sign, and the evaluation with a modified Rankin Scale score. Each patient also benefited from an ophthalmologic examination, including a visual acuity examination, a Lancaster test, a funduscopy, and a Goldman test.

\section{Analysis of the Dural Arteriovenous Anatomy}

Each patient underwent a 6-vessel pretherapeutic DSA under local anesthesia. We reviewed these examinations with attention paid to the location of the fistulous point and angiographic factors that could explain the ocular sign (venous reflux, compression of a cranial nerve, or venous congestion). The presence of a venous thrombosis, stenosis, or ectasia was also noted. Special attention was paid to the pathophysiology of ocular signs presented by each patient.

\section{Endovascular Treatment}

All endovascular treatment was performed with the patient under general anesthesia. After cerebral MR imaging and DSA were per- 
formed, the location and the anatomy of the dAVF were discussed, with the aim of choosing the more appropriate treatment.

\section{Postoperative Follow-Up}

Follow-up started at the time of the last embolization session and finished with the last visit or angiography. Angiographic follow-up was performed 6 months after treatment to confirm the

\section{Table 1: Demographic and clinical data of the population}

\begin{tabular}{lc}
\hline \multicolumn{1}{c}{ Variable } & Patients $(\boldsymbol{n}=\mathbf{1 3})$ \\
\hline Age (yr) (median) (range) & $50.3(15-72)$ \\
Men & $9(69.2 \%)$ \\
Clinical signs & \\
Pulsatile tinnitus & $5(38.5 \%)$ \\
Chemosis & $8(61.5 \%)$ \\
Exophthalmia & $5(38.5 \%)$ \\
Loss of visual acuity & $5(38.5 \%)$ \\
Ocular hypertension & $1(7.7 . \%)$ \\
Oculomotor palsy & $4(30.8 \%)$ \\
Third CN palsy & $2(15.4 \%)$ \\
Fourth CN palsy & $2(15.4 \%)$ \\
Sixth CN palsy & $3(23.1 \%)$ \\
Papillary edema & $9(69.2 \%)$ \\
Time between first sign and diagnosis (mo) & $10(1-36)$ \\
mRS score before treatment & \\
1 & $9(69.2 \%)$ \\
2 & $4(30.8 \%)$ \\
\hline
\end{tabular}

Note:- $\mathrm{CN}$ indicates cranial nerve.
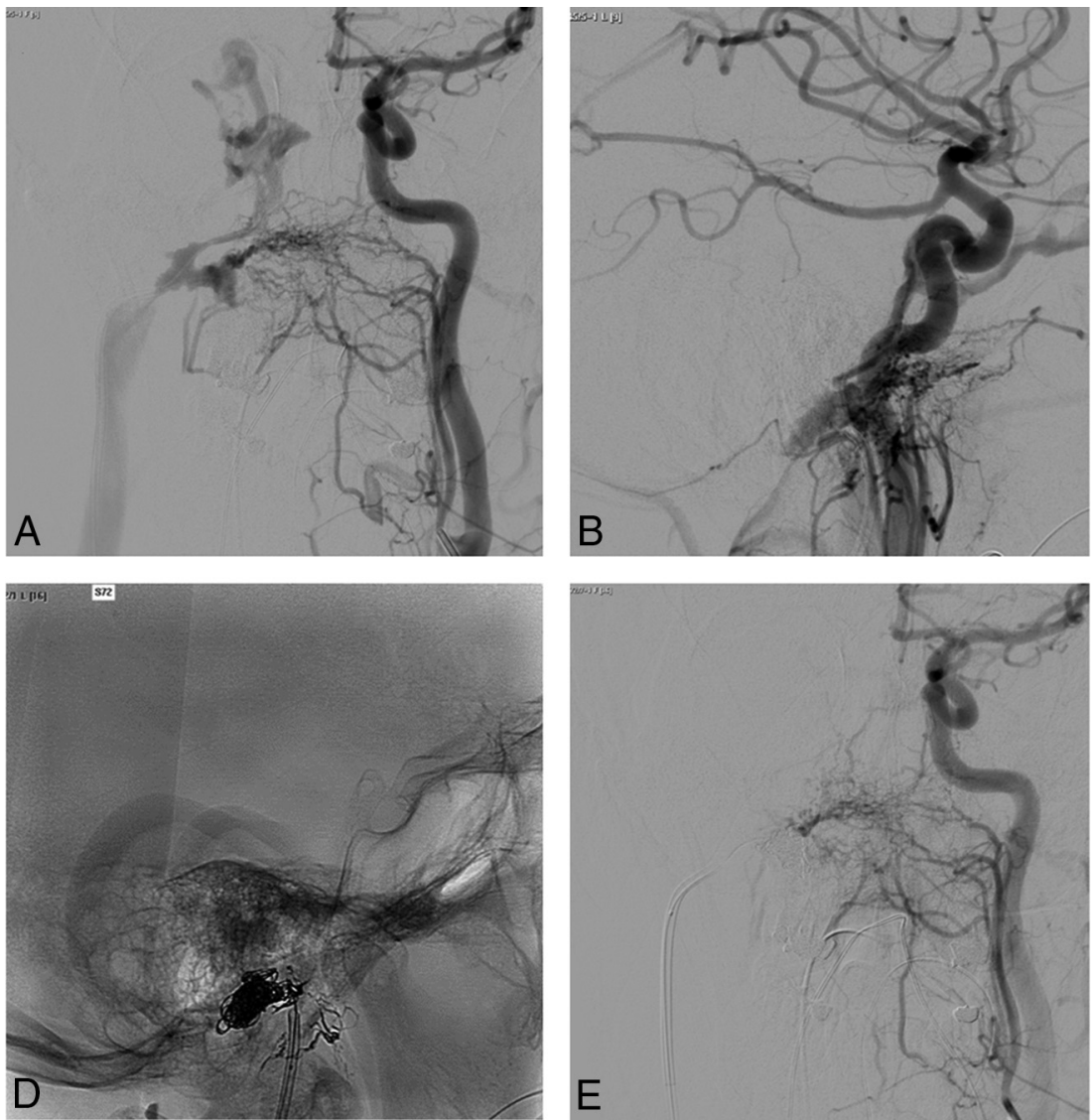

occlusion of the arteriovenous shunt. A neurologic examination with evaluation with the mRS was systematically performed for each visit. An ophthalmologic examination was performed in the posttreatment period (within 3 months after each embolization session).

\section{RESULTS}

\section{Demographic Data and Clinical Presentation}

Between 2000 and 2015, 13 patients met the inclusion criteria of this study. Patient baseline data and clinical signs are described in Table 1 . The mean age was 50.3 years (range, 15-72 years) with a male preponderance $(\mathrm{M} / \mathrm{F}$ ratio $=2.25$ and 2:25). The most common clinical sign was chemosis in 8 patients $(61.5 \%)$, followed by loss of visual acuity ( 5 patients, $38.5 \%$ ), exophthalmia (5 patients, $38.5 \%)$, and ocular hypertension in 1 patient (7.7\%). Papillary edema was noted in 9 patients $(69.2 \%)$ and was unilateral in 7 cases and bilateral in 2 others.

\section{Local Venous Reflux into the Ophthalmic Veins}

Ocular signs could be explained by ophthalmic venous engorgement secondary to venous reflux from the fistulous point in most cases (7/13) in our series. All patients in this group presented with a chemosis, which was associated with proptosis in 5 patients, progressive loss of acuity in 1 patient, ocular hypertension in 1 patient, and oculomotor nerve paresis in 2 patients. The classifi-

FIG 1. Pretherapeutic left common carotid artery DSA in anteroposterior $(A)$ and lateral $(B)$ projections and external carotid artery DSA in a lateral projection $(C)$ highlighting a right jugular foramen dAVF with venous reflux into the right inferior petrosal sinus, the right cavernous sinus, and the right superior ophthalmic vein in a patient presenting with right chemosis and exophthalmia. D, Note the cast of Onyx (Covidien, Irvine, California) after an arterial embolization. Posttherapeutic left common carotid injections in anteroposterior $(E)$ and lateral $(F)$ projections. 

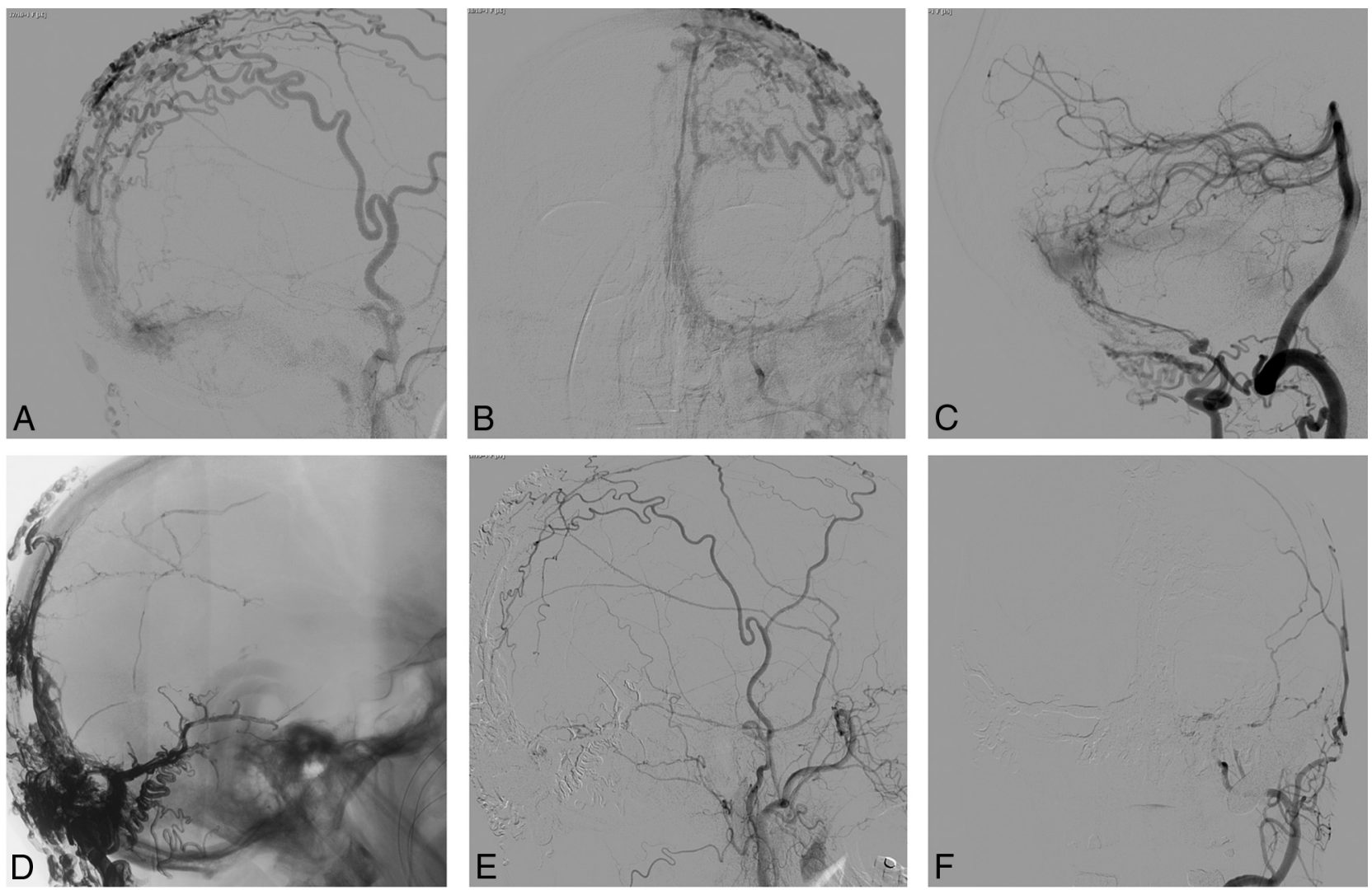

FIG 2. Lateral $(A)$ and anteroposterior (B) projections of a pretherapeutic left external carotid artery DSA showing a complex fistula of the posterior third of the superior sagittal sinus in a patient with signs of intracranial hypertension. Lateral projection (C) of the left vertebral artery injection in the same patient shows multiple fistulous points on the transverse and sigmoid sinuses. $D, A$ lateral skull $\mathrm{x}$-ray with the important cast of Onyx used to treat the fistulas. Posttherapeutic lateral $(E)$ and anteroposterior $(F)$ projections of the left external carotid artery injection.

cation of Lariboisière was $2 \mathrm{a}+\mathrm{b}$ in 4 cases and 3 in 3 cases. Three fistulas were located in the middle cranial fossa, with venous drainage involving the sphenoparietal sinus, the cavernous sinus, and the superior ophthalmic vein (SOV). The venous reflux also involved the uncal vein in 1 of these cases. Two other fistulas were located in the lateral sinus with a venous reflux important enough to involve the vein of Labbé, the superficial middle cerebral vein, the cavernous sinus, and the SOV. The last 2 fistulas were located at the jugular foramen, and the venous reflux involved the inferior petrosal sinus, the cavernous sinus, and, finally, the SOV (Fig 1).

\section{Intracranial Hypertension due to Venous Reflux}

In this series, 4 patients had signs of intracranial hypertension syndrome, in particular a progressive decrease of visual acuity associated with headache ( 1 case) and sixth nerve palsy ( 1 case). In all cases, a bilateral papillary edema was found at funduscopy. All these patients had high-flow dAVFs with bilateral venous reflux and type $2 \mathrm{a}+\mathrm{b}$ fistulas. The fistulous point was always located on a large venous sinus: the rectus sinus in 1 case, lateral sinuses in 2 cases, and the superior sagittal sinus in 1 case (Fig 2). Arterial feeders of these fistulas did not present particularity instead of their high number (4-12) and the recruitment of transosseous branches from occipital and superficial temporal arteries. In 3 of these 4 cases, multiple fistulous points were identified along the sinus wall. In 1 case, a sinus thrombosis, which increases venous reflux, was present. None of these cases had reversal flow of the
SOV, and the ocular signs could not be explained by local orbital venous obstruction.

\section{Cranial Nerve Compression by a Venous Ectasia}

In 2 patients in this series, the ocular signs could only be explained by cranial nerve compression. The first one was a dAVF located on the free border of the tentorium. Feeding arteries were the medial tentorial artery (meningohypophyseal trunk), the petrous branch of the middle meningeal artery, and the mastoid branch of the occipital artery. The venous drainage interested the basal vein of Rosenthal without venous reflux but with a venous ectasia of the posterior third of the basal vein of Rosenthal (Lariboisière fistula type 4). This patient was admitted for a progressive trochlear nerve palsy, which could easily be explained by the compression of the trochlear nerve by the dilated basal vein of Rosenthal in the ambient cistern (Fig 3).

The second case was a man who had a 1-month history of progressive unilateral oculoparesis. The ophthalmologic examinations revealed third, fourth, and sixth cranial nerve paresis. The DSA showed a dAVF located under the lesser sphenoid wing in the region of the superior orbital fissure. Arterial feeders were the recurrent meningeal artery, the middle meningeal artery, and the deep temporal artery. Venous drainage involved the SOV and the cavernous sinus, with a venous ectasia of the SOV in the superior orbital fissure (Lariboisière fistula type 4). This venous ectasia was the only explanation for the oculomotor paresis. 

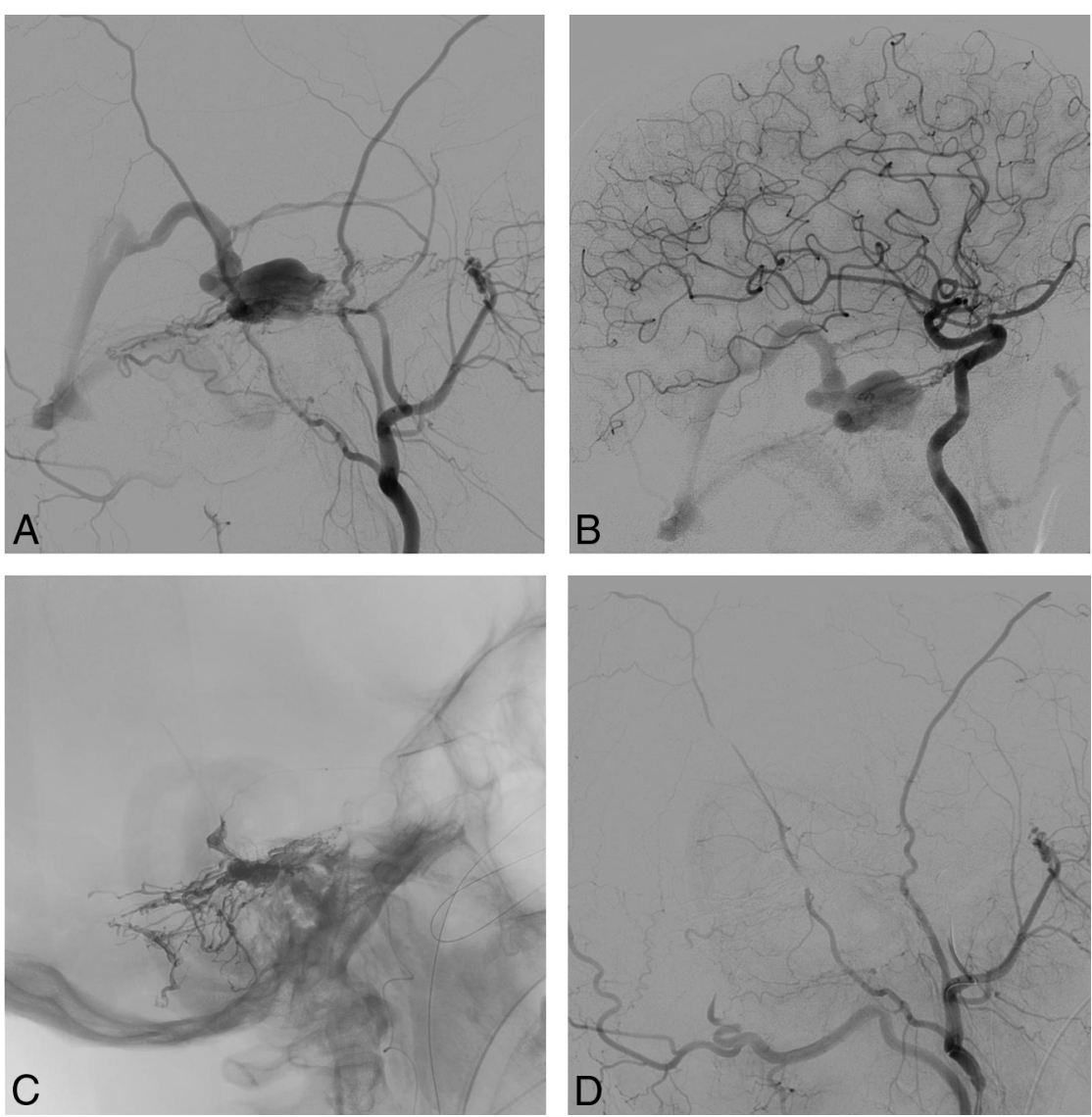

FIG 3. Pretherapeutic lateral projections of the right external $(A)$ and internal $(B)$ carotid artery DSA highlighting a tentorial dAVF with venous ectasia of the third portion of the basal vein in a patient with a trochlear nerve deficit. C, Note the cast of Onyx (Covidien, Irvine, California) after injection through the middle meningeal artery branches. $D$, Posttherapeutic right external carotid artery DSA in a lateral projection without a residual fistula.

\section{Treatment of the Pathology}

Treatment details and outcome are summarized in Table 2. Every patient was treated by endovascular therapy. Twenty-three embolization sessions were necessary to treat the fistulas. Seven patients were successfully treated in 1 session, 4 patients had 2 sessions, and 2 fistulas needed 3 sessions to be completely obliterated. One patient was treated by microsurgical exclusion of a middle cranial fossa fistula after failed embolization.

An arterial approach was used in 8 sessions; a venous approach, in 14 patients; and a combined approach, in 1 patient. The overall success rate per embolization session was 52.2\% (12 embolization sessions). In 4 patients (15.4\%), the fistula persisted after the injection of the embolic agent, and in 2 patients (7.7\%), the venous access to the fistula was impossible. Among these 4 patients, in 2, a partial embolization was planned to reduce the venous reflux and to decrease the intracranial pressure. In 2 patients $(8.8 \%)$, the implantation of coils in an arterial feeder near the fistulous point was sufficient to obliterate the fistula.

\section{Clinical and Angiographic Follow-Up}

The fistula was cured at the 6-month follow-up DSA in 11 cases (78.5\%). A partial embolization was planned for 2 patients who had a complex dAVF and intracranial hypertension.

Neurologic complications were found after 2 embolization sessions. One patient with a tentorial fistula developed an exten- sive venous thrombosis a few hours after the embolization, responsible for a hemiplegia and multiple cranial nerve palsies. This patient was dependent and died 1 year after the treatment. The other patient with a complication had a middle cranial fossa dAVF treated by coils and Glubran Tiss (Aspide Medical, La Talaudiére, France). Twenty-four hours after the treatment, she developed a temporal lobe hematoma with a contralateral hemiparesis (mRS 3). The mean clinical follow-up was 10.1 months (range, 1-48 months). At the last follow-up visit, 3 patients had $\mathrm{mRS}$ $0 ; 6, \mathrm{mRS} 1 ; 2, \mathrm{mRS} 2 ; 1, \mathrm{mRS} 3$; and 1 , mRS 6 . The ophthalmologic examination findings 3 months after treatment were normal in 8 patients.

\section{DISCUSSION}

Cranial dural arteriovenous fistulas are often symptomatic, with a pulsatile tinnitus with or without headache, focal neurologic deficit, or compressive signs. ${ }^{1,3,6}$ The occurrence of an intracerebral hematoma is also a well-known entity, in particular in cases of venous reflux, cortical vein involvement, or venous ectasia. ${ }^{1}$ In rare cases, a dAVF could be symptomatic by ocular signs such as chemosis, exophthalmia, loss of visual acuity, or oculomotor nerve palsy. ${ }^{5}$ The literature of these dAVFs is exclusively represented by case reports and small case series. ${ }^{3,16,19}$ These articles generally described the symptoms and angiographic anatomy of the fistula well but detailed analysis of the pathophysiology was always missed except for the series of Cognard et al. ${ }^{4}$

The first and most frequent type of fistula in our series occurred when the ocular signs could be explained by a direct venous reflux into the orbital veins. This was also the most frequent type in the literature. ${ }^{7}$ These fistulas are generally located on the transverse sinus, ${ }^{8}$ with a reflux through the vein of Labbé or at the foramen magnum. For a venous reflux to produce orbital venous hypertension, thrombosis or noncompetence of a large venous collector (jugular bulb or transverse sinus) is necessary. The middle cranial fossa is also described with reflux into the sphenoparietal sinus. ${ }^{3}$ The anatomic variant of the paracavernous sinus could be an important factor in venous reflux into the superior orbital vein. Two case reports ${ }^{9,10}$ of tentorial dAVFs with venous reflux into a basal vein, an uncal vein, and a superior ophthalmic vein are also described, but they seem to be rare. The importance of the venous reflux and the high frequency of venous ectasia in these fistulas are 2 reasons for aggressive management. These fistulas were treated by an arterial approach in all cases described. In the literature and in our series, ocular symptoms and signs were reversible after endovascular treatment of the fistula. With a sim- 
Table 2: Details of endovascular treatment and outcome

\begin{tabular}{lc}
\hline \multicolumn{1}{c}{ Variable } & No. (\%) \\
\hline Total No. of embolization sessions & 23 \\
Embolization per patient (mean) (range) & $1.8(1-3)$ \\
Venous approach & $14(60.8 \%)$ \\
Arterial approach & $8(34.8 \%)$ \\
Combined approach & $1(4.3 \%)$ \\
Overall success rate & $12(52.2 \%)$ \\
Incomplete closed fistula & $4(15.4 \%)$ \\
Impossible to catheterize & $2(7.7 \%)$ \\
Embolic agent used & \\
Onyx & $12(52.2 \%)$ \\
Coils + Onyx & $5(21.7)$ \\
Glubran Tiss & $4(17.4 \%)$ \\
Coils & $2(8.8 \%)$ \\
Success rate & \\
By patient & $11 / 14(78.5 \%)$ \\
Associated microsurgery & $1 / 14(7.2 \%)$ \\
Complications & \\
Permanent & $2(8.7 \%)$ \\
Death & $1(4.3 \%)$ \\
Follow-up & \\
Mean (range) (mo) & $10.1(1-48)$ \\
Last mRS & \\
0 & $3(23.1 \%)$ \\
1 & $6(46.2 \%)$ \\
2 & $2(15.4 \%)$ \\
3 & $1(7.2 \%)$ \\
6 & $1(7.2 \%)$ \\
Ophthalmologic follow-up & $8(61.5 \%)$ \\
Normal findings & $1(7.2 \%)$ \\
Central scotoma & $1(7.2 \%)$ \\
Third and sixth nerve palsy & $1(7.2 \%)$ \\
Ocular hypertonia & $1(7.2 \%)$ \\
Persistent papillary edema & $1(7.2 \%)$ \\
Lost to follow-up & \\
\hline & \\
&
\end{tabular}

ilar pathologic mechanism, few cases of cerebral arteriovenous malformations ${ }^{11}$ with ocular signs were reported.

Intracranial hypertension syndrome could also explain the ocular signs. Patients had a loss of visual acuity secondary to papillary edema or optic disc atrophy. ${ }^{1,12,13}$ Generally, these dAVFs were multiple arteriovenous shunts located on large sinuses, important because they could influence the venous drainage of the encephalon. ${ }^{4}$ The venous engorgement or reflux provoked by the high flow of the fistula causes hydrocephalus and possibly parenchymal edema. The first article describing an elevation of intracranial pressure in a posterior fossa dAVF was that of Lamas et $\mathrm{al}^{14}$ in 1977. In 1998, Cognard et $\mathrm{al}^{4}$ published a series including 13 patients with dAVFs with signs of intracranial hypertension. Among these 13 patients, 8 had a papilledema and 4 had optic disc atrophy. Other clinical signs described were seizures, tinnitus, headache, and diplopia.

As for patients in our series, the location of the fistula was always on a large dural sinus with multiple arteriovenous shunts. We found a sinus anomaly (thrombosis, agenesis) in 7 of 13 patients. The treatment of this type of fistula must be aggressive to avoid the progression of intracranial hypertension signs and irreversibility of optic disc atrophy. This treatment is always challenging because of the multiplicity of shunts. Partial embolization could be an alternative to decrease the flow of the pathology without risking occlusion of a large dural sinus. This partial treatment is often temporary. An aggressive arterial embolization of the fistulas could be performed with concomitant inflation of a large balloon into the dural sinus, but this treatment exposes the patient to a higher risk of complications. ${ }^{15}$ The placement of a ventriculoperitoneal shunt is also a palliative solution. The choice of treatment depends on the anatomy of the arteriovenous shunts, their location, the severity of the clinical signs, and the patient's comorbidities.

The compression of a cranial nerve by venous dilation in case of arteriovenous shunt is well-known, especially for the trigeminal nerve. The occurrence of an oculomotor paresis secondary to the same diagnosis is rarely reported in the literature, perhaps due to lack of knowledge of anatomic details. Only 1 case report ${ }^{16}$ of a trochlear nerve deficit caused by a tentorial dAVF and, in particular, a venous ectasia could be found in the literature. The anatomic details and the relationship between the trochlear nerve and the venous structures in the perimesencephalic cisterns were not well-developed in this article. In our series, a similar case is presented. This is a dAVF located in the posterior incisural space with its venous drainage involving the posterior part of the basal vein. As described by Ono et $\mathrm{al}^{17}$ and by Joo and Rhoton, ${ }^{18}$ the trochlear nerve has an intimal relationship with the third portion of the basal vein and with the superior cerebellar artery into the ambient cistern. A dilation of the basal vein in its ambient course could easily result in trochlear nerve compression and a dilation of the lateromesencephalic vein. The other case in our series with the same pathologic mechanism is a dAVF located in the region of the superior orbital fissure with drainage into the sphenoparietal sinus and the superior ophthalmic vein. This latter vein had an important dilation at its intracanalicular portion that caused a compression of nerves (third, fourth, and sixth nerves associated with an ophthalmic hypoesthesia, typical of superior orbital fissure syndrome).

Another type of fistula with ocular signs is an intraorbital fistula, with direct drainage into the superior ophthalmic vein. We did not have fistulas of this type in our series, but in 2 case reports, ${ }^{7,19}$ they are well-documented. These fistulas were fed by ethmoidal branches and were directly drained by the superior ophthalmic vein; this scenario created intraorbital hypertension. One of these 2 cases was successfully treated by arterial embolization $^{7}$; the other, by a venous approach after surgical exposure of the superior ophthalmic vein. Pan et $\mathrm{al}^{5}$ also reported 3 other cases of intraorbital shunt treated by radiosurgery, but not enough details were reported to understand the pathophysiology of these fistulas.

\section{CONCLUSIONS}

DAVFs with ocular signs could be classified into 4 types due to the pathologic mechanisms that explain these signs. The first type is a local venous reflux into the superior ophthalmic vein, the second is a massive venous engorgement of the cerebrum responsible for intracranial hypertension, the third is a direct compression of an oculomotor nerve by a venous dilation, and the last is an intraorbital fistula with direct drainage into the superior ophthalmic vein.

Disclosures: Michel Piotin-UNRELATED: Consultancy: Medtronic,* Stryker,* MicroVention, * Balt*; Payment for Lectures (including service on Speakers Bureaus): Penumbra. * Money paid to the institution. 


\section{REFERENCES}

1. Awad IA, Little JR, Akarawi WP, et al. Intracranial dural arteriovenous malformations: factors predisposing to an aggressive neurological course. J Neurosurg 1990;72:839-50 Medline

2. Cognard C, Gobin YP, Pierot L, et al. Cerebral dural arteriovenous fistulas: clinical and angiographic correlation with a revised classification of venous drainage. Radiology 1995;194:671-80 Medline

3. Hayreh SS. Ocular Vascular Occlusion Disorders. New York: Springer; 2015

4. Cognard C, Casasco A, Toevi M, et al. Dural arteriovenous fistulas as a cause of intracranial hypertension due to impairment of cranial venous outflow. J Neurol Neurosurg Psychiatry 1998;65:308-16 Medline

5. Pan HC, Sun $\mathrm{MH}$, Chen $\mathrm{WH}$, et al. Minimally invasive approaches to treating chemosis of the eyes from unusual dural arteriovenous fistulae. Minim Invasive Neurosurg 2009;52:222-28 CrossRef Medline

6. Gandhi D, Chen J, Pearl M, et al. Intracranial dural arteriovenous fistulas: classification, imaging findings, and treatment. AJNR Am J Neuroradiol 2012;33:1007-13 CrossRef Medline

7. Inoue $\mathrm{A}$, Tagawa $\mathrm{M}$, Kumon $\mathrm{Y}$, et al. Ethmoidal dural arteriovenous fistula with unusual drainage route treated by transarterial embolization. J Neurointerv Surg 2015;7:e15 CrossRef Medline

8. Feyissa AM, Ponce LL, Patterson JT, et al. Dural arteriovenous fistula presenting with exophthalmos and seizures. J Neurol Sci 2014; 338:229-31 CrossRef Medline

9. Benndorf G, Schmidt S, Sollmann WP, et al. Tentorial dural arteriovenous fistula presenting with various visual symptoms related to anterior and posterior visual pathway dysfunction: case report. Neurosurgery 2003;53:222-26; discussion 226-27 Medline

10. Iizuka Y, Maehara T, Hishii M, et al. Successful transarterial glue embolisation by wedged technique for a tentorial dural arteriovenous fistula presenting with a conjunctival injection. Neuroradiology 2001;43:677-79 Medline

11. Gregory ME, Berry-Brincat A, Ghosh YK, et al. An arteriovenous malformation masquerading as a carotid-cavernous sinus fistula. Am J Ophthalmol 2005;140:548-50 Medline

12. Biousse V, Mendicino ME, Simon DJ, et al. The ophthalmology of intracranial vascular abnormalities. Am J Ophthalmol 1998;125; 527-44 Medline

13. Gelwan MJ, Choi IS, Berenstein A, et al. Dural arteriovenous malformations and papilledema. Neurosurgery 1988;22:1079-84 Medline

14. Lamas E, Lobato RD, Esperarza J, et al. Dural posterior fossa AVM producing raised sagittal simus pressure: case report. J Neurosurg 1977;46:804-10 Medline

15. Zako M, Murata K, Inukai T, et al. Long-term progressive deterioration of visual function after papilledema improved by embolization of a dural arteriovenous fistula in the sigmoid sinus: a case report. J Med Case Rep 2014;8:392 CrossRef Medline

16. Mariniello G, Briganti F, Vergara P, et al. Dural tentorial arteriovenous fistula causing isolated trochlear nerve palsy: remission after endovascular embolization. J Neurointerv Surg 2012;4:e5 CrossRef Medline

17. Ono M, Rhoton AL Jr, Peace D, et al. Microsurgical anatomy of the deep venous system of the brain. Neurosurgery 1984;15:621-57 Medline

18. Joo W, Rhoton AL Jr. Microsurgical anatomy of the trochlear nerve. Clin Anat 2015;28:857-64 CrossRef Medline

19. Daou B, Chalouhi N, Williams K, et al. An unusual case of an ethmoidal arteriovenous fistula draining into the superior ophthalmic vein. Neurosurgery 2015 Aug 6. [Epub ahead of print] Medline 